\title{
A Robust Approach for Low Resolution Medical Image Enhancement using DTCWT and SVD
}

\author{
${ }^{\text {"1M.Mahesh }}{ }^{2}$ T. Murali Krishna, ${ }^{3}$ B.Naresh Babu \\ 1, 2, 3 Department of ECE, S V Engineering College For Women, Tirupathi
}

Received: 06th October 2017 Accepted: 14th November 2017, Published: 31st December 2017

\begin{abstract}
Low Resolution Image Enhancement is one of the most important features in Image processing domain whose main target is to improve with the help of which contrast and the visual appearance of an image can be improved that makes the input image more suitable than the original one for specific application. Low Resolution Medical Image enhancement is the active research field. A novel approach to enhance Low resolution medical images is combination of Dual tree complex wavelet transform(DTCWT), A Non-Local Mean(NLM) filter and singular value decomposition (SVD).Here, SVD is proposed to enhance the contrast of an image while high frequency sub bands are produced using dual-tree complex wavelet transform. A NLM Filter is used to remove the artifacts produced by dual-tree complex wavelet transform. Interpolated filtered high frequency sub-bands and contrast enhanced low resolution image are combined using inverse dual-tree complex wavelet transform to obtain contrast enhanced image of super resolution. Qualitative and quantitative measures such as Peak Signal to Noise Ratio (PSNR), Mean Square Error and Mean Absolute Error are used to justify the importance of proposed technique.
\end{abstract}

Keywords: Image Enhancement, DTCWT, SVD, NLM, MSE, MAE, PSNR

\section{Introduction}

Low Resolution enhancement of medical images has been a matter of concern and a very attractive field for the researchers as well. As analysis of medical images mainly depends on the size and quality of acquired image like noise immunity, illumination conditions etc [1]. For proper diagnosis the interpretation of medical images and its resolution enhancement are very much desirable. Therefore many different techniques has been proposed for medical image resolution enhancement (MIRE)[2]. There has been a lot of research mainly on frequency domain methods. After the fast decomposition method given by Mallat [3], Wavelet Transform emerged as a new time -frequency analysis tool which accomplished desired results in image processing field. Many image enhancement methods were developed based on wavelet transform such as Lu et al[4], Yang and Hansell [5], Fang and Qi

[6], Zhou et al [7] etc. General approach of image enhancement lies in getting detail information of image through wavelet transform by decomposing high frequency sub-images to get enhanced image of better visual quality.

As our visual system is very much sensitive to contrast, for surgeons and physicians in addition to $\mathrm{RE}$ (to assist interpolation and diagnosis) contrast enhancement (CE) is also equally important and desirable. In image processing applications generally most effective and simplest. In [8] using un-decimated decomposition was used in stationary wavelet transform approach which outperforms other conventional wavelet techniques.

Discrete wavelet transform (DWT) in combination with SWT and nonlocal means filter is used in [9]. As visual system of human being is more sensitive to contrast therefore enhancement is also important for physician and surgeons in addition to other image processing techniques to for diagnosis purpose. The local histogram equalization and generalized histogram equalization methods are also used for contrast enhancement in many image processing applications. Intensity information of an image is contained in singular values [11] and good contrast image can be obtained by changing the singular value. In $[12,13]$ Image enhancement has been done using Singular value decomposition which has proved better than conventional techniques i.e. histogram based approaches. In [14] Discrete wavelet transform is used for contrast enhancement. Least squares trained filters are used in [15] for image resolution enhancement. The method proposed in [16] shows impressive results but this technique uses only training process not temporal which is also one of the important factors for image quality enhancement.

In this paper a new scheme has been proposed for resolution enhancement using a hybrid approach comprising dual-tree complex wavelet transform and Singular value decomposition followed by filtering where a low resolution input image is first enhanced using dual-tree complex wavelet transform (DTCWT), NLM and SVD. Here, DTCWT is used to preserve the general properties for computational efficiency and perfect reconstruction with well-balanced frequency responses [17]. The artifacts created by DTCWT are less than those produced due to DWT after the modification done in coefficients and hence provide better results [18]. Also NLM filter is 
provided to make some improvement in dual-tree complex wavelet transform resolution enhancement.

In proposed scheme an automatic calculation is proposed to have the value of weights which would be used for training process. Since the singular values have the image intensity information by which modification of singular value is done to produce desired contrast in the image. The superiority of proposed technique can be observed quantitatively as well as visually.

\section{Material and Method}

The high frequency components of an image i.e. edges are much vulnerable to blurring effect and low resolution therefore it becomes essential to preserve high components of the image during image enhancement. In the given method, First, Complex Wavelet Transform of level 1 is applied to input bio medical image. The weight coefficient based on histogram equalized input image and input image is calculated using the following formula

$$
\mathrm{W}=\frac{\max \left(S_{1}\right)+\max \left(S_{2}\right)}{2 \times \max \left(S_{1}\right)}
$$

Where S2 is Singular Value Decomposition of the histogram equalized image and S1 is Singular Value Decomposition of the input image.

Then the calculated weight coefficient is applied to get SVD Equalized image

$$
\mathbf{L}_{\mathrm{e}}=\mathbf{U}_{1} * \mathbf{S}_{\mathrm{W}} * \mathbf{V}_{\mathbf{1}}^{\mathrm{T}}
$$

Where $\mathrm{U}_{1}$ and $\mathrm{V}_{1}$ are orthogonal square matrices, $L_{e}$ is enhanced low frequency sub-image $S_{w}$ is given as follows

$$
\mathbf{S}_{\mathbf{w}}=\mathrm{W} * \mathrm{~S}_{1}
$$

The above SVD Equalized image is used along with the low frequency coefficients for inverse complex wavelet transform to get the image with contrast enhancement.

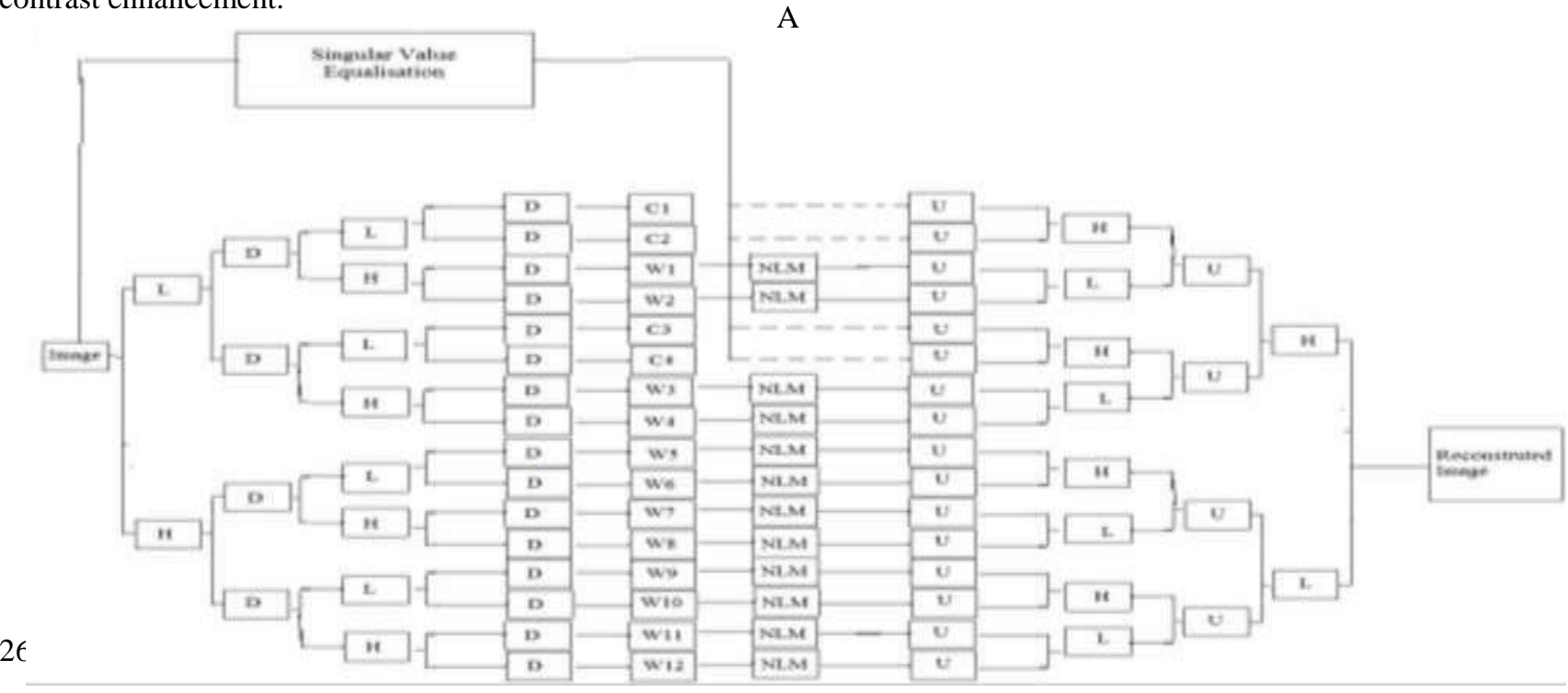

Figure 1 shows the architecture of proposed system. where block labeled as D represents Down sampling operation, block $U$ indicates up sampling operation, block L stands for low frequency sub images and bock $\mathrm{H}$ shows high frequency respectively.C1, C2, C3 and $\mathrm{C} 4$ are those image coefficients which have been produced by low pass filtering and $\mathrm{W} 1, \mathrm{~W} 2, \mathrm{~W} 3 \ldots . . \mathrm{W} 12$ are the wavelet coefficients that have been produced by high pass filtering. NLM indicates Non-local means filter which works on the basis of self-similarity approach.

\section{Flow chart}




A

A

A

Fig 1: Mechanism of image enhancement using Proposed Method

\section{Results and Discussion}

The proposed system has been tested and verified on various medical images to show the superiority of the proposed system in terms of visual and numerical results over existing system. By considering the commonly used quantitative measures like, Mean-Squared Error (MSE), Mean Approximate Error (MAE) and Peak Signal-to-
Noise Ratio (PSNR) performance of proposed method can be evaluated MSE gives the squared error between the enhanced and the original image, Mean Absolute Error between two digital images represents the absolute closeness of these images to each other whereas PSNR is the ratio between the maximum possible value of a pixels of image to the power of distorting noise. Fig. 2(a, b), Fig. 3(a, b), and Fig. 4(a, b) are shown below in that fig. (a) is original image, (b) is high contrast image obtained from proposed method. Quantitative analysis of proposed technique has been shown in Table I

Fig. 2: First medical image and respective processing on the same:

a) Original Image (b) DTCWT-SVD Enhanced Image

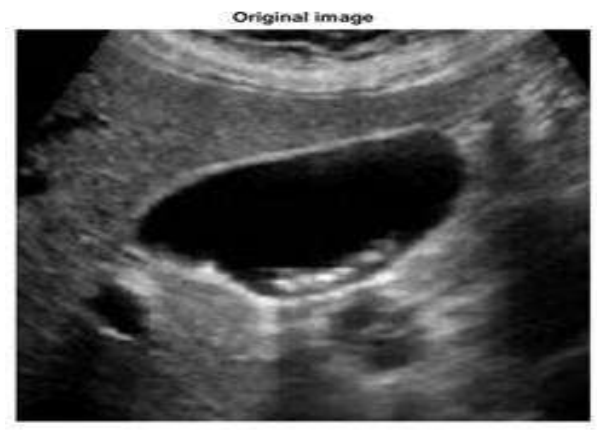

(a)

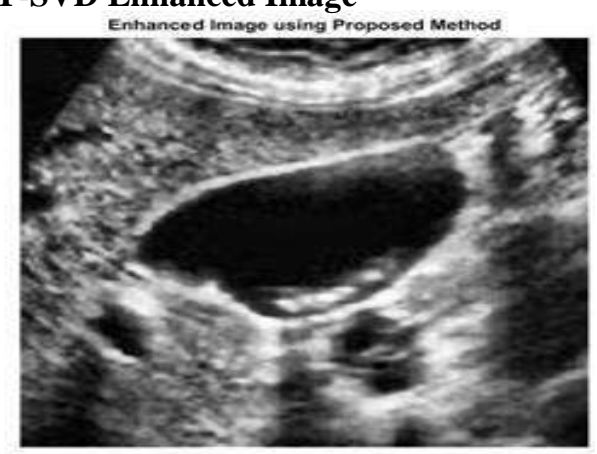

(b)

Fig. 3: Second medical image and respective processing on the same: (a) Original Image (b) DTCWT-SVD Enhanced Image

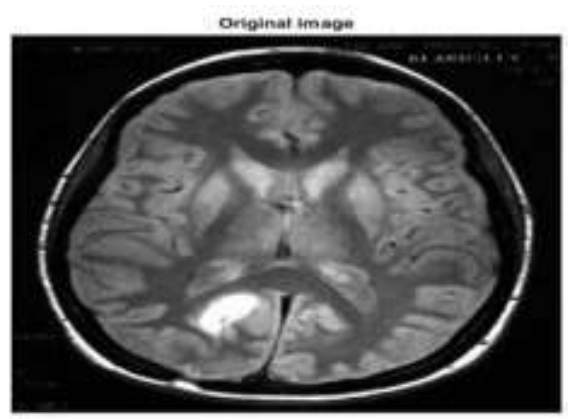

(a)

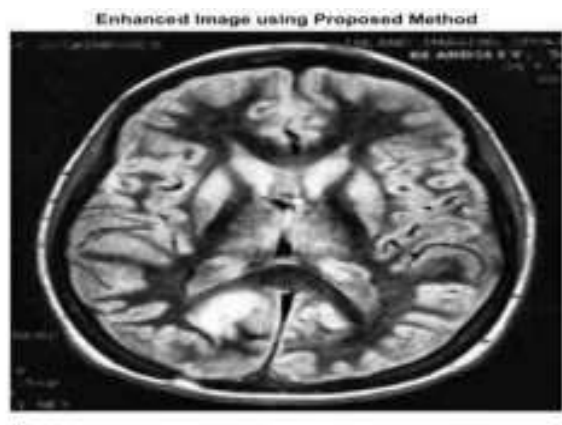

(b)

Fig. 4: Third medical image and respective processing on the same:

(a) Original Image (b) DTCWT-SVD Enhanced Image

Table I. Calculated performance parameters of enhanced medical images

\begin{tabular}{|c|c|c|c|}
\hline Image & PSNR & MSE & MAE \\
\hline 1 & 39.9095 & 0.0013264 & 0.02395 \\
\hline 2 & 47.2991 & 0.0036772 & 1.005 \\
\hline 3 & 34.9585 & 0.0011255 & 2.100 \\
\hline
\end{tabular}

\section{Conclusion}

The proposed algorithm is a hybrid approach using DTCWT, NLM filter and SVD and has been tested on a set of medical images. The medical input image is decomposed using DTCWT. Less artifacts are

generated with the help of DTCWT compared to that of DWT because of nearly shift-invariance characteristic of DTCWT. Further image quality is improved using NLM filtering approach and SVD is

used for to get originality of image and obtain a better quality image both quantitatively and qualitatively. Simulation results showed that proposed technique outperforms other conventional 
techniques for improving visual quality of medical images for proper manual interpretation and Computer based diagnosis. This can be considered as an efficient method which will be very useful in some important areas for image enhancement in satellite imaging, remote imaging, medical imaging etc

\section{References}

[1] Gagandeep Kaur ,Shruti Vashist,"A Robust Approach for Medical image Enhancement using DTCWT", International Journal of Computer Applications (0975 - 8887) Volume 167 - No.6, June 2017

[2] R C Gonzalez, R. E. Woods, "Digital Image Processing Using MATLAB”, $2^{\text {nd }}$ ed, Prentice Hall

[3] Mallat, S.G.: 'Multifrequency channel decompositions of image and wavelet models', IEEE Trans. Acoust. Speech Signal Process., 1989, 37, (12), pp. 20912110 .

[4] Lu, J., Healy, D.M., and Weaver, J.B.: „Contrast enhancement of medical images using multi-scale edge repressentation ${ }^{\text {ee }}$, Opt. Eng., 1994, 33, (7), pp. 2151-2161.

[5] Yang, G. , and Hansell, D. M. : 'CT image enhancement with wavelet analysis for the detection of small airways disease', IEEE Trans. Med. Imaging, 1997, 16, (6), pp. 953961.

[6] Zhou, X., Zhou, S., Huang, F., and Zhou, X.T.: „New algorithm of image enhancement based on wavelet transforme ${ }^{e}$, Comput. Appl., 2005, 25, (3),pp. 606-608.

[7] W.L. Lee, C.C. Yang, H.T. Wu, M.J. Chen, Wavelet-based interpolation scheme for resolution enhancement of medical images, J. Signal Process. Syst. 55 (2009) pp.251-265.

[8] M.Z. Iqbal, A. Ghafoor, A.M. Siddiqui, Wavelet transform and non local means based super resolution, in: International Symposium.

[9] H. Demirel, C. Ozcinar, G. Anbarjafari, Satellite image contrast enhancement using discrete wavelet transform and singular value decomposition, IEEE Geosci. Remote Sens. Lett. 7 (April (2)) (2010).

[10] Y. Tian, T. Tan, Y. Wang, Y. Fang, Do singular values contain adequate information for face recognition, Pattern Recognit. 36 (3) (2003) pp. 649-655.

[11]H. Demirel, G. Anbarjafari, M.N.S. Jahromi, Image equalization based on singular value decomposition, in: IEEE International
Symposium on Computer and Information Sciences, October 27-29, 2008, pp. 1-5.

[12]Fang, Y., and Qi, F.: „A method of wavelet image enhancement based on soft threshold ${ }^{\text {ee }}$, Comput. Eng. Appl., 2002, 23, pp. 16-19.

[13] H. Demirel, G. Anbarjafari, M.N.S. Jahromi, Image equalization based on singular value decomposition, in: IEEE International Symposium on Computer and Information Sciences, October 27-29, 2008, pp. 1-5.

[14] Y. Yang, Z. Su, L. Sun, Medical image enhancement algorithm based on wavelet transform, Electron. Lett. 46 (January (2)) (2010).

[15] J.L. Starck, F. Murtagh, J.M. Fadili, Sparse Image and Signal ProcesQ5 sing: Wavelets, Curvelets, Morphological Diversity, Cambridge University Press, 2010.

[16] L. Shao, R. Yan, X. Li, Y. Lin, From heuristic optimization to dictionary learning: a review and comprehensive comparison of image denoising algorithm, IEEE Trans. Cybern. (2013).

[17] Khare, A., Khare, M., Jeong, Y., Kim, H., and Jeon, M.: 'Despeckling ofmedical ultrasound images using daubechies complex wavelet transform', Signal Process., 2010, pp. 428 439.

[18] Nick Kingsbury,"Shift invariant properties of the dual tree Complex Wavelet Transform" IEEE Trans. On signal processing letters, 1999. 